\title{
Measurement and relationship between critical tube diameter and critical energy for direct blast initiation of gaseous detonations
}

\author{
Bo Zhang ${ }^{1}$, Hoi Dick Ng ${ }^{2 *}$, John H.S. Lee ${ }^{3}$ \\ ${ }^{1}$ Beijing Institute of Technology \\ State Key Laboratory of Explosion Science and Technology, Beijing, 100081, China \\ ${ }^{2}$ Concordia University \\ Department of Mechanical and Industrial Engineering \\ Montreal, H3G 1M8, Canada \\ ${ }^{3}$ McGill University \\ Department of Mechanical Engineering, Montreal, H3A 2K6, Canada
}

\section{Research Highlights}

1. New measurement of detonation dynamic parameters in common hydrocarbon mixtures

2. Study the scaling between critical tube diameter, initiation energy and cell size

3. Assess models for direct link between critical tube diameter and initiation energy

4. Experimental data useful for the explosion safety assessment in industrial setting 


\title{
Measurement and relationship between critical tube diameter and critical energy for direct blast initiation of gaseous detonations
}

\author{
Bo Zhang ${ }^{1}$, Hoi Dick Ng ${ }^{2 *}$, John H.S. Lee ${ }^{3}$ \\ ${ }^{1}$ Beijing Institute of Technology \\ State Key Laboratory of Explosion Science and Technology, Beijing, 100081, China \\ (Currently at: School of Resources and Environmental Engineering, East China University of Science and \\ Technology, Shanghai, 200237, China) \\ ${ }^{2}$ Concordia University \\ Department of Mechanical and Industrial Engineering \\ Montreal, H3G 1M8, Canada \\ ${ }^{3}$ McGill University \\ Department of Mechanical Engineering, Montreal, H3A 2K6, Canada
}

*Corresponding Author

Department of Mechanical and Industrial Engineering

Concordia University

1455 de Maisonneuve Blvd. West

Montréal, H3G 1M8, Canada

e-mail: hoing@encs.concordia.ca

Tel.: (514) 848-2424 (ext. 3177)

Fax: (514) 848-3175

Revised manuscript submitted to Journal of Loss Prevention in Process Industries

April, 2013 


\title{
Measurement and relationship between critical tube diameter and critical energy for direct blast initiation of gaseous detonations
}

\begin{abstract}
For the explosion safety assessment in industrial setting, detonation dynamic parameters provide important information on the sensitivity and conditions whereby detonations can be favorably occurred. In this study, new measurement of the critical tube diameter and the critical energy for direct initiation of a detonation is reported for a number of hydrocarbon-oxygen mixtures. The simultaneous experimental measurement carried out in this work allows the investigation of the direct scaling between these two dynamic parameter quantities of gaseous detonations. Using the new set of data, this paper also assesses the validity of an existing semi-empirical initiation model, namely, the surface energy model by Lee, and a simplified work done model. Both phenomenological models provide a general relationship between the two dynamic detonation parameters and comparison shows a good agreement between the theoretical results and the experimental measurement. The scaling of critical tube diameter with detonation cell size in this study also confirms the results in the previous literature.
\end{abstract}

Keywords: detonation hazards; detonation dynamic parameters; critical tube diameter; critical energy; cell size; direct initiation 


\section{Introduction}

Accidental explosions are key concern for all combustible fuels related to process safety. Accidental explosions and detonations occur often in industry and result in casualty and severe loss of property at industrial facilities (e.g., Gao and Hirano 2006; Hirano 2001; Johnson 2010; Wen et al. 2011). For preventive measures of these accidents and the design of effective mitigation scheme, it requires a realistic assessment of explosion hazards of combustible fuels. Accumulation of detonation dynamic parameter data such as cell size, initiation energy, critical tube diameter or detonability limit for various combustible systems and the ability to predict these quantities not only are of great fundamental significance to further the understanding of the detonation wave structure and its propagation, but also provide useful information for safety assessment and evaluation of potential hazards of explosive mixtures (Lee 1984; Matsui and Lee 1978; Ng and Lee 2008). In this study, two particular detonation dynamic parameters, namely the critical tube diameter and critical energy of direct initiation are studied in detail. The critical tube diameter, $d_{c}$, is the minimum tube diameter from which an emergent planer detonation wave can transmit into free space without failure. Critical initiation energy, $E_{\mathrm{c}}$, refers to the minimum energy required for the direct blast initiation of a spherical detonation in a given explosive mixture.

From the dimensional analysis consideration, it is possible to realize that once a characteristic length scale is determined, the various dynamic parameters can be readily correlated with that particular length scale. Indeed, there are numerous studies attempted to link the characteristic detonation cell size $\lambda$ - the average width of the cellular or fish-like 
pattern measured from smoked foils - as the fundamental chemical length scale, to the critical tube diameter, initiation energy as well as detonation limits (Lee et al. 1982). However, a quantitative theory for predicting the cell size is still lacking and experimental determination of a unique characteristic cell size from smoked foils can be difficult and often very subjective due to the inherent instability of the detonation front and cellular irregularity in common explosive mixtures (Lee 2008).

Beside the characteristic cell size $\lambda$, critical tube diameter $d_{\mathrm{c}}$ can be considered as an alternative length scale for various correlations. It can be argued that direct experimental measurement of critical tube diameter is perhaps more trustworthy than the determination of cell size. In fact, many initiation models such as the work done concept and the surface energy model are developed based on the relationship linking the cell size to the critical energy via the critical tube diameter (Lee 1984, 1997; Benedick et al. 1986). Therefore, one should verify the direct link between the initiation energy and the critical tube diameter without evaluation of the cell size. The critical tube diameter phenomenon may also provide a better problem from the modeling point of view compared to a quantitative theory of cell size prediction.

In this paper, new measurement of critical tube diameter and critical energy for direct initiation of spherical detonation are reported. Unlike cell size data which are abundant in the literature but yet possess a significant degree of uncertainty, data on critical tube diameters for a wide range of hydrocarbon gaseous mixtures are relatively scarce. Therefore, these new measurements provide addition to the detonation database and give further useful information 
for the explosion safety assessment in industrial setting. In this study, the critical tube diameters experiments with five different tube diameters scales, $D$, were performed for a number of different explosive mixtures at various initial conditions. In addition, the purpose of this work is the simultaneous determination of critical tube diameter and initiation energy for direct blast initiation of detonations in these different mixtures. The results then allow one to verify directly the scaling relationship between these two dynamic parameters. In fact, comparison and validation of detonation initiation models are often made between the measured values of the critical initiation parameters with those predicted with cell sizes as input parameters following the classical empirical relationships $\left(d_{\mathrm{c}}=13 \lambda\right.$ and $\left.E_{c} \sim \rho_{o} V_{C J}^{2} \lambda^{2}\right)$; and large discrepancies often obtained due to the uncertainty in cell size measurement and in some special mixtures such as highly argon diluted mixtures, the breakdown of the classical empirical correlation (Desbordes et al. 1993). Here, the direct scaling between the critical tube diameter and initiation energy is reported and verified through the use of two semi-empirical initiation models without the recourse to the cell size measurement.

\section{Experimental details}

The direct measurement of the critical tube diameter and the critical energy has been carried out in a number of common combustible mixtures (i.e. $\mathrm{C}_{2} \mathrm{H}_{2}-\mathrm{O}_{2}, \mathrm{C}_{2} \mathrm{H}_{2}-2.5 \mathrm{O}_{2}, \mathrm{C}_{2} \mathrm{H}_{2}-4 \mathrm{O}_{2}$, $\left.\mathrm{C}_{2} \mathrm{H}_{4}-3 \mathrm{O}_{2}, \mathrm{C}_{3} \mathrm{H}_{8}-5 \mathrm{O}_{2}, \mathrm{C}_{2} \mathrm{H}_{2}-2.5 \mathrm{O}_{2}-50 \% \mathrm{Ar}, \mathrm{C}_{2} \mathrm{H}_{2}-2.5 \mathrm{O}_{2}-70 \% \mathrm{Ar}\right)$. The schematic of the critical tube diameter experiment is shown Fig. 1. The detonation is first initiated by a high-voltage spark ignition source at the top of the vertical circular steel tube and exits into a large 
spherical bomb chamber at the other end. A photo probe and a shock pin are mounted at the top and bottom of the spherical bomb, which are used to determine the time-of-arrival signal of the wave.

Typical traces for a surviving diverging detonation wave, i.e., for $d>d_{\mathrm{c}}$ and a detonation failure case in a stoichiometric $\mathrm{C}_{2} \mathrm{H}_{2}-2.5 \mathrm{O}_{2}$ mixture with the tube diameter of $19.05 \mathrm{~mm}$ and initial pressures of $p_{\mathrm{o}}=12 \mathrm{kPa}$ and $11 \mathrm{kPa}$ are shown in Fig. 2 and Fig. 3, respectively. It can be seen from Fig. 2 that at an initial pressure of $12 \mathrm{kPa}$, the arrival time of the expanding wave is $201 \mu \mathrm{sec}$ when it reaches the photo probe and $317 \mu \mathrm{sec}$ at the shock pin. The velocity of the wave is $2073.4 \mathrm{~m} / \mathrm{s}$ and $2136.7 \mathrm{~m} / \mathrm{s}$ in the vertical tube and spherical chamber, which is of $91.1 \%$ and $94.4 \%$ of $\mathrm{CJ}$ detonation velocity, respectively. It shows that at an initial pressure of $12 \mathrm{kPa}$, the tube diameter is above the critical value, thus the planar detonation can successfully transit into a spherical detonation. While for an unsuccessful transmission when $d<d_{\mathrm{c}}$, Fig. 3 shows that when the initial pressure decreases to $11 \mathrm{kPa}$, although a detonation wave propagates in the vertical tube at a velocity around $90 \% \mathrm{CJ}$ detonation velocity, the detonation fails after exiting into the free space and the velocity of the expanding wave is only $23.6 \%$ of the $\mathrm{CJ}$ velocity value. For each successful and unsuccessful initiation of spherical detonation at least 3 shots are repeated to confirm the critical pressure that can form a spherical detonation at each tube diameter. In some experiments, the tube inner diameters are also varied via inserting smaller diameter tubes.

Using the same spherical bomb setup and by removing the vertical circular tube as shown in Fig. 1, experiments are also carried out to determine the critical initiation energy 
using the same mixtures and under the same initial conditions considered for the critical tube diameter problem. The procedure to distinguish detonation initiation and details to estimate the actual spark discharge energy from the ignition system can be found in authors' previous studies (Kamenskihs et al. 2010; Zhang et al. 2011a, 2011b, 2012b).

\section{Results and discussion}

The critical tube diameter for various mixtures as a function of initial pressure obtained by experiment is shown in Fig. 4. Other correlations for undiluted mixtures, which are based on the experimental data measured by Matsui and Lee (1978), are also included for comparison, represented by the solid lines in the plots. It can be seen from Fig. 4 that for the undiluted mixtures, the experimental data from this study is in good agreement with those found in Matsui and Lee (1978). For the stoichiometric acetylene-oxygen diluted with 50\% and 70\% argon mixtures, there was no previous data to compare. Nevertheless, from the experimental trend it shows that with the increasing amount of argon dilution, the initial pressure at which the planar detonation can transmit into spherical detonation increases consequently using the same tube diameter. In other words, with more argon dilution, the mixtures tend to be less sensitive to form a spherical detonation when the planar detonation emerges from the tube into unconfined space.

Similar to many previous studies, it is worth to correlate the present critical tube diameter results with available detonation cell size data tabulated in CALTECH Detonation Database (Kaneshige and Shepherd 1997). The curve fit correlations of available cell size data 
as a function of initial pressure for the mixtures considered in this study are given in Table 1. By comparing the critical tube diameter and cell size as shown in Fig. 5, it is found for the undiluted mixtures (i.e., $\mathrm{C}_{2} \mathrm{H}_{2}-\mathrm{O}_{2}, \mathrm{C}_{2} \mathrm{H}_{2}-2.5 \mathrm{O}_{2}, \mathrm{C}_{2} \mathrm{H}_{2}-4 \mathrm{O}_{2}, \mathrm{C}_{2} \mathrm{H}_{4}-3 \mathrm{O}_{2}, \mathrm{C}_{3} \mathrm{H}_{8}-5 \mathrm{O}_{2}$ ) the relationship between these two parameters is $d_{\mathrm{c}} \approx 13 \lambda$. This observation is in good agreement with previous investigations (Mitrofanov and Soloukhin 1965; Knystautas et al. 1982). Similar to previous results obtained by many researchers (Desbordes et al. 1993; Moen et al. 1986; Sherpherd et al. 1986), the present experiment data also confirms that for mixtures highly diluted with argon, the $d_{c} \approx 13 \lambda$ correlation breaks down. It is found that the proportional factor equals 21 and 29 for the stoichiometric acetylene-oxygen mixtures with $50 \%$ and $70 \%$ argon dilution, respectively. Detonations in highly argon diluted mixtures are stable and their propagation mechanism is different from that of cellular detonations in unstable mixtures (Radulescu 2003; Lee 1996), thus the failure and re-initiation of a diffracting stable detonation emerging from a tube into unconfined space are also different, resulting in the breakdown of $d_{c} \approx 13 \lambda$.

Although a number of semi-empirical models to predict the critical initiation energy can be found in literature (Benedick et al. 1986; Vasil'ev 2012), here we consider two existing initiation models of which the energy formulations are obtained from the analogy of the critical tube diameter problem in term of $d_{c}$, the diameter above which the plane detonation wave can successful transform into a spherical one when it exits the tube. This in essence assumes the energy released by the planar detonation tube is comparable to the initiation energy of a spherical detonation (Sochet et al. 1999). The selected models hence allow us to 
explore readily the direct link between the critical tube diameter and the critical energy for direct initiation. First, with the knowledge of experimentally measured critical tube diameters at different initial pressures, the critical energy can be theoretically estimated by the surface energy model proposed by Lee (1997). The surface energy model is a semi-empirical phenomenological model that relates the point blast initiation mode with the planar wave initiation mode. The link is established based on the minimum surface energy of the critical tube to the surface area of the critical size of the minimum detonation kernel; in other words, the correlation is through equating the surface energy contained in the wave in both cases at criticality. Thus the surface energy contained in the point blast initiated spherical detonation at the time when the wave has decayed to the CJ state from its overdriven state is equivalent to the energy in the planar detonation wave in the critical tube diameter situation. This formulation leads to the following equation for estimating the critical initiation energy (Zhang et al. 2011a):

$$
E_{c}=4 \pi \gamma p_{o} M_{C J}^{2} I\left(\frac{d_{C}}{4}\right)^{3}
$$

In this study, a simplified work done model is also investigated (Matsui and Lee 1978; Desbordes 1988; Sochet et al. 1999; Zhang et al. 2012a). Assuming the energy needed to re-initiate a detonation downstream of the unconfined space in the critical tube diameter problem can be related to the work done delivered by the detonation product in the confined tube (i.e., a fictitious piston) over a period $t^{*}$, the energy can be obtained by: 


$$
E_{c}=\int_{0}^{t *} p_{C J} \frac{\pi d_{c}^{2}}{4} u_{C J} d t
$$

where $p_{\mathrm{CJ}}$ and $u_{\mathrm{CJ}}$ denote the $\mathrm{CJ}$ detonation pressure and particle velocity, respectively. $t^{*}$ can be modeled as the time when the rarefaction wave reaches the tube axis, which can be approximately by $t^{*} \sim d_{\mathrm{c}} / 2 a_{C J}$ with $a_{\mathrm{CJ}}$ being the sound speed of the detonation products (Vasil'ev 1998). Therefore, a simplified work done model expression can be given as:

$$
E_{c}=\frac{p_{C J} u_{C J} \pi d_{c}^{3}}{8 a_{C J}}
$$

In general, although the surface energy model and simplified work done model are established from a different phenomenological consideration, both can provide direct link between critical tube diameter and critical energy for direct initiation. However, the previous use of these two theories often included the empirical correlation between the cell size and critical tube diameter (i.e., $d_{\mathrm{c}}=13 \lambda$ ) due to the wider availability of the cell size data. Therefore, the validity of these two initiation models in common hydrocarbon-oxygen-argon mixtures was never assessed before with values of critical tube diameter as direct inputs.

As an example, the theoretical prediction and the experimentally measured critical energy as a function of initial pressure for the equimolar $\mathrm{C}_{2} \mathrm{H}_{2}-\mathrm{O}_{2}$ mixture is shown in Table 2. From the comparison, it is found that the theoretical critical energies obtained from both models are very close to the experimental data. Comparing the theoretical and experimental critical energies of the other mixtures which are shown in Fig. 6 and Fig. 7, similar scenario can be found in general. From these figures, however, it appears that the results for $\mathrm{C}_{2} \mathrm{H}_{2} / \mathrm{O}_{2}$ based mixtures agree best with the surface energy model. This is in accord with the literature that 
among different modeling approaches to estimate critical energy, the "surface energy" model agrees well with available experimental data on direct initiation (Benedick et al. 1993; Zhang et al. 2012a; Vasil'ev and Vasil'ev 2010). For these mixtures, the results also indicate that the simplified work done model predicts higher value of critical initiation energy. The work done or piston model is based on the detonation products playing a determining role in initiating the spherical detonation wave. However, physically the work is done not exactly by the products of the plane detonation wave but by the decelerated gas of the curved front through a critical thickness from the critical point (Bannikov \& Vasil'ev 1993). It is thus not surprising that the simplified work done model always estimates a higher $E_{\mathrm{c}}$ than the surface energy model. Another interesting observation can be seen from Fig. 6 d) and e) which appear to show a different behavior in the results. For $\mathrm{C}_{2} \mathrm{H}_{4}-3 \mathrm{O}_{2}$ and $\mathrm{C}_{3} \mathrm{H}_{8}-5 \mathrm{O}_{2}$ mixtures, the detonation wave is typically more unstable compared to $\mathrm{C}_{2} \mathrm{H}_{2}-\mathrm{O}_{2}$ based mixtures in the sense that the detonation cellular front is more irregular with larger degree of instabilities. The deviation of the measured initiation energy from the prediction using the surface energy model can therefore be attributed to the important of detonation instability. According to Lee's hypothesis (1996), the critical tube diameter phenomenon in highly irregular mixtures is related to detonation instability where the failure of the diverging detonation wave is due to the suppression of instability at the front during the front expansion. Therefore a more unstable mixtures with large degree of instability (or in which the impacts of transverse waves is significant in re-initiating the mixtures downstream) may enhance the transmission of the detonation from a confined tube to the open space (Lee 1996; Jones et al. 1996). However, for direct initiation of 
detonation, experimental results show that the early development and successful initiation originate from an overdriven detonation with very fine cell structure (Lee 2008) and hence, the inherent detonation instability may play a less important role in defining direct initiation. In summary, due to the multidimensional instability effect within the propagating detonation cellular front in the irregular mixtures, the energy required in re-initiating and propagating a spherical detonation in the critical tube diameter problem may deviate from the one equivalent for successful direct blast initiation from a point source. It is also important to note that the two initiation models considered here used the critical tube diameter as an equivalent to the critical initiation energy $E_{\mathrm{c}}$. In the critical tube diameter phenomenon, the region where energy is evolved has a finite size and thus, it does not exactly satisfy the condition for an ideal source in the direct initiation phenomenon (Bannikov \& Vasil'ev 1993). The important role of detonation instability which can play a leading role in the detonation transmission is also not considered. From these models and dimensional consideration, the critical energy is proportional to the cube of characteristic size given here by the critical tube diameter (i.e., $E_{\mathrm{c}}$ $\left.\sim d_{c}{ }^{3}\right)$ and therefore, any associated experimental uncertainty in the critical tube diameter are raised to power of 3 in the energy estimation. Evidently, better model can be developed by refining the criterion within the framework of using $d_{\mathrm{c}}$ as an equivalent to the critical initiation energy $E_{\mathrm{c}}$, see Vasil'ev (2012). Therefore, taking all these aspects into consideration, the maximum $\%$ deviation from all the experimental results $(51 \%$ for the surface energy model and $92.4 \%$ for the work done model) seems reasonable and that both the surface energy model and the simplified work done model provide equally well the direct link between the 
critical tube problem and direct blast initiation of gaseous detonation with reasonable accuracy.

\section{Concluding remarks}

In this paper, new data on the critical tube diameters and critical energies for direct blast initiation of spherical detonations in different mixtures (i.e., $\mathrm{C}_{2} \mathrm{H}_{2}-\mathrm{O}_{2}, \mathrm{C}_{2} \mathrm{H}_{2}-2.5 \mathrm{O}_{2}, \mathrm{C}_{2} \mathrm{H}_{2}-4 \mathrm{O}_{2}$, $\mathrm{C}_{2} \mathrm{H}_{4}-3 \mathrm{O}_{2}, \mathrm{C}_{3} \mathrm{H}_{8}-5 \mathrm{O}_{2}, \mathrm{C}_{2} \mathrm{H}_{2}-2.5 \mathrm{O}_{2}-50 \% \mathrm{Ar}, \mathrm{C}_{2} \mathrm{H}_{2}-2.5 \mathrm{O}_{2}-70 \% \mathrm{Ar}$ ) are reported. These results obtained from simultaneous experimental measurement allow the analysis of any scaling and relationship between cell size, critical tube diameter and critical energy. The present results are first validated that, for the undiluted mixtures the critical tube diameter is found to be around 11-14 times of the characteristic cell size, which agrees with the well-known empirical correlation of $d_{\mathrm{C}} \approx 13 \lambda$. The experimental data once again show that the $13 \lambda$ behavior does not apply for those mixtures which are highly diluted with argon. The proportional factor is found to be $21 \lambda$ and $29 \lambda$ for the stoichiometric acetylene-oxygen mixtures with $50 \%$ and $70 \%$ argon dilution, respectively. These results agree with previous hypothesis (Lee 1996) that the failure and re-initiation of a diffracting stable detonation emerging from a tube into unconfined space are different, resulting in the breakdown of $d_{c} \approx 13 \lambda$ correlation. The comparison between the theoretical predicted critical energy, from surface energy model and simplified work done model, and the experimental data shows a very good agreement. Both theoretical models appear to provide a direct link between critical tube diameter and critical initiation energy from phenomenological consideration. In conclusion, these results and 
models should found applications in safety hazard assessment of these fuels and in developing new predictive tools and preventive measures against accidental explosions causing severe damages.

\section{Acknowledgement}

This work is supported by the Natural Sciences and Engineering Research Council of Canada (NSERC) and the Fonds de recherche du Québec - Nature et technologies.

\section{References}

Bannikov N.V. and Vasil'ev A.A. (1993) Plane initiation of a detonation. Combust. Expl. Shock Waves 29(3): 409-414.

Benedick W.B., Guirao C.M., Knystautas R. and Lee J.H.S. (1986) Critical charge for the direct initiation of detonation in gaseous fuel-air mixtures. Prog. Astronaut. Aeronaut. 106:181-202.

Desbordes D. (1988) Transmission of overdriven plane detonations: critical diameter as a function of cell regularity and size. Prog. Astro. Aero. 11: 170-185.

Desbordes D., Guerraud C., Hamada L. and Presles H.N. (1993) Failure of classical dynamic parameters relationships in highly regular cellular detonation systems. Prog. Astronaut. Aeronaut. 153: 347.

Gao L. and Hirano T. (2006) Process of accidental explosions at a refuse derived fuel storage. J. Loss Prevention Proc. Ind. 19:288-291.

Hirano T. (2001) Methodology for case studies of accidental gas explosion. J. Loss Prevention Proc. Ind. 14:553-557.

Johnson D.M. (2010) The potential for vapour cloud explosions - lessons from the Buncefield accident. J. Loss Prevention Proc. Ind. 23(6):921-927. 
Jones D.A., Kemister G., Oran E.S., Sichel M. (1996) The influence of cellular structure on detonation transmission. Shock Waves 6:119-129.

Kamenskihs V., Ng H.D. and Lee J.H.S. (2010) Measurement of critical energy for direct initiation of spherical detonations in high-pressure $\mathrm{H}_{2}-\mathrm{O}_{2}$ mixtures. Combust. Flame 157(9): 1795-1799.

Kaneshige M. and Shepherd J.E. (1997) Detonation database, GALCIT Technical Report FM97-8.

Knystautas R., Lee J.H. and Guirao C. (1982) The critical tube diameter for detonation failure in hydrocarbon-air mixtures. Combust. Flame 48: 63-83.

Lee J.H.S. (1984) Dynamic parameters of gaseous detonations. Ann. Rev Fluids Mech. 16: 311-36.

Lee J.H.S. (1996) On the critical tube diameter. In: Bowen, J. (ed.) Dynamics of Exothermicity, pp. 321 Gordon and Breach, Amsterdam.

Lee J.H.S. (1997) Initiation of gaseous detonation. Annu. Rev. Phys. Chem. 28:75-104.

Lee J.H.S. (2008) The Detonation Phenomenon. Cambridge University Press.

Lee J.H.S, Knystautas R. and Guirao C.M. (1982) The link between cell size, critical tube diameter, initiation energy and detonability limits. In Fuel-Air Explosions, U. Waterloo Press, p. $157-187$.

Matsui H. and Lee J.H.S. (1978) On the measure of the relative detonation hazards of gaseous fuel-oxygen and air mixture. Proc. Combust. Inst. 17: 1269.

Mitrofanov V.V. and Soloukhin R.I. (1965) The diffraction of multifront detonation waves. Sov. Phys.-Dokl. 9(12): 1055.

Moen I., Sulmistras A., Thomas G.O., Bjerketvedt D. and Thibault P.A. (1986) Influence of cellular regularity on the behavior of gaseous detonations. Prog. Astronaut. Aeronaut. 106: 220.

$\mathrm{Ng}$ H.D. and Lee J.H.S. (2008) Comments on explosion problems for hydrogen safety. J. Loss Prevention in the Proc. Ind. 21(2):136-146.

Radulescu M.I. (2003) The Propagation and Failure Mechanism of Gaseous Detonations: Experiments in Porous-Walled Tubes. Ph.D. thesis, McGill University, Canada.

Shepherd J.E., Moen I., Murray S. and Thibault P.I. (1986) Analyses of the cellular structure of detonations. Proc. Combust. Inst. 21: 1649. 
Sochet I., Lamy T., Brossard J., Vaglio C. and Cayzac R. (1999) Critical tube diameter for detonation transmission and critical initiation energy of spherical detonation. Shock Waves 9: 113-123.

Vasil'ev A.A. (1998) Diffraction estimate of the critical energy for initiation of gaseous detonation. Combust. Expl. Shock Waves 34(4): 433-437.

Vasil'ev A.A. (2012) Dynamic parameters of detonation. In: F. Zhang, Editor, Shock Waves Science and Technology Library, Vol 6: Detonation Dynamics, Springer, Berlin Heidelberg (2012) Chap. 4.

Vasil'ev A.A. and Vasil'ev V.A. (2010) Diffraction of waves in combustible mixtures. J. Eng. Phys. Thermophys. 83(6): 1178-1196.

Wen J.X., Heidari A., Ferraris S. and Tam V.H.Y. (2011) Numerical simulation of propane detonation in medium and large scale geometries. J. Loss Prevention Proc. Ind. 24:187-193.

Zhang B., Kamenskihs V., Ng H.D. and Lee J.H.S. (2011a) Direct blast initiation of spherical gaseous detonation in highly argon diluted mixtures. Proc Combust. Inst. 33(2): 2265-2271.

Zhang B., Ng H.D., Mével R. and Lee J.H.S. (2011b) Critical energy for direct initiation of spherical detonations in H2/N2O/Ar mixtures. Int. J. Hydrogen Energy 36: 5707-5716.

Zhang B., Ng H.D. and Lee, J.H.S. (2012a) Measurement of effective blast energy for direct initiation of spherical gaseous detonations from high-voltage spark discharge. Shock Waves 22(1):1-7.

Zhang, B., Ng, H.D. and Lee, J.H.S. (2012b) Measurement and scaling analysis of critical energy for direct initiation of detonation. Shock Waves 22(3): 275-279. 


\section{List of tables}

Table 1. Cell size correlations for mixtures as a function of initial pressure given by $\lambda[\mathrm{mm}]=C\left(P_{0}[\mathrm{KPa}]\right)^{-\alpha}$

Table 2. Theoretical and experimental critical energy for equimolar $\mathrm{C}_{2} \mathrm{H}_{2}-\mathrm{O}_{2}$ at different initial pressure

\begin{tabular}{ccc}
\hline Mixtures & $C$ & $\alpha$ \\
\hline $\mathrm{C}_{2} \mathrm{H}_{2}-\mathrm{O}_{2}$ & 9.2382 & 0.9625 \\
$\mathrm{C}_{2} \mathrm{H}_{2}-2.5 \mathrm{O}_{2}$ & 26.262 & 1.1889 \\
$\mathrm{C}_{2} \mathrm{H}_{2}-4 \mathrm{O}_{2}$ & 54.967 & 1.1656 \\
$\mathrm{C}_{2} \mathrm{H}_{4}-3 \mathrm{O}_{2}$ & 56.458 & 0.9736 \\
$\mathrm{C}_{3} \mathrm{H}_{8}-5 \mathrm{O}_{2}$ & 186.55 & 1.1729 \\
$\mathrm{C}_{2} \mathrm{H}_{2}-2.5 \mathrm{O}_{2}-50 \% \mathrm{Ar} *$ & 61.5 & 1.12 \\
$\mathrm{C}_{2} \mathrm{H}_{2}-2.5 \mathrm{O}_{2}-70 \% \mathrm{Ar}^{*}$ & 113.8 & 1.20 \\
\hline
\end{tabular}

* From Radulescu (2003)

Table 1.

\begin{tabular}{cccccc}
\hline Mixture & $\begin{array}{c}D_{\mathrm{C}} \\
(\mathrm{mm})\end{array}$ & $\begin{array}{c}p_{0} \\
(\mathrm{kPa})\end{array}$ & $\begin{array}{c}E(\mathrm{~J}) \\
(\text { Surface Energy model })\end{array}$ & $\begin{array}{c}E(\mathrm{~J}) \\
(\text { Work done model) }\end{array}$ & $\begin{array}{c}E(\mathrm{~J}) \\
(\text { Experimental })\end{array}$ \\
\hline & 19.05 & 6 & 0.34 & 0.57 & $0.36-0.41$ \\
$\mathrm{C}_{2} \mathrm{H}_{2}-\mathrm{O}_{2}$ & 16.1 & 8 & 0.28 & 0.46 & $0.28-0.32$ \\
& 12.97 & 10 & 0.18 & 0.31 & $0.17-0.20$ \\
& 6.6 & 19 & 0.05 & 0.08 & $0.04-0.06$ \\
\hline
\end{tabular}

Table 2. 


\section{Figure captions}

Fig. 1. Schematic of the experimental setup for the critical tube diameter measurement.

Fig. 2. Arrival time trace of a planar detonation emerging into an unconfined space:

successful initiation of a spherical detonation for $\mathrm{C}_{2} \mathrm{H}_{2}-2.5 \mathrm{O}_{2}$ mixture at an initial pressure of $12 \mathrm{kPa}$.

Fig. 3. Arrival time trace of a planar detonation emerging into an unconfined space:

unsuccessful initiation of a spherical detonation for $\mathrm{C}_{2} \mathrm{H}_{2}-2.5 \mathrm{O}_{2}$ mixture at the initial pressure of $11 \mathrm{kPa}$.

Fig. 4. The variation of critical tube diameter as a function of initial pressure.

Fig. 5. Critical tube diameter and cell size as a function of initial pressure for a) $\mathrm{C}_{2} \mathrm{H}_{2}-2.5 \mathrm{O}_{2}$;
b) $\mathrm{C}_{2} \mathrm{H}_{2}-4 \mathrm{O}_{2}$; c) $\mathrm{C}_{2} \mathrm{H}_{2}-\mathrm{O}_{2}$; d) $\mathrm{C}_{2} \mathrm{H}_{4}-3 \mathrm{O}_{2}$; e) $\mathrm{C}_{3} \mathrm{H}_{8}-5 \mathrm{O}_{2}$; f) $\mathrm{C}_{2} \mathrm{H}_{2}-2.5 \mathrm{O}_{2}-50 \%$ Ar; and g)
$\mathrm{C}_{2} \mathrm{H}_{2}-2.5 \mathrm{O}_{2}-70 \%$ Ar mixtures.

Fig. 6. Critical energy as a function of critical tube diameter for a) $\mathrm{C}_{2} \mathrm{H}_{2}-\mathrm{O}_{2}$; b) $\mathrm{C}_{2} \mathrm{H}_{2}-2.5 \mathrm{O}_{2}$; c)

$$
\mathrm{C}_{2} \mathrm{H}_{2}-4 \mathrm{O}_{2} ; \text { d) } \mathrm{C}_{2} \mathrm{H}_{4}-3 \mathrm{O}_{2} \text {; and e) } \mathrm{C}_{3} \mathrm{H}_{8}-5 \mathrm{O}_{2} \text { mixtures. }
$$

Fig. 7. Critical energy as a function of critical tube diameter for a) $\mathrm{C}_{2} \mathrm{H}_{2}-2.5 \mathrm{O}_{2}-50 \% \mathrm{Ar}$; b)

$$
\mathrm{C}_{2} \mathrm{H}_{2}-2.5 \mathrm{O}_{2}-70 \% \text { Ar. }
$$


Fig. 1

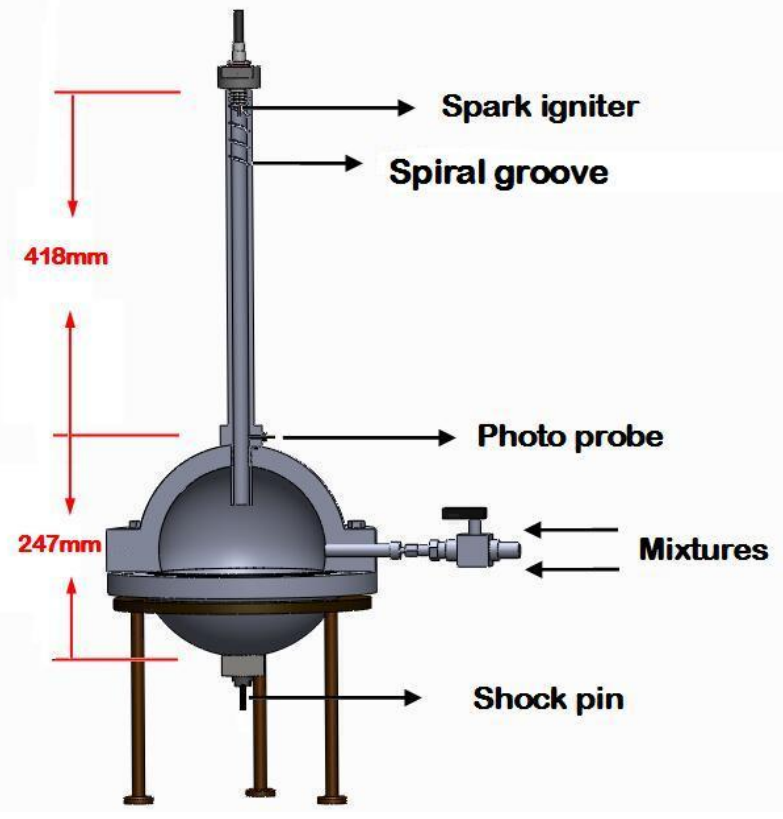


Fig. 2

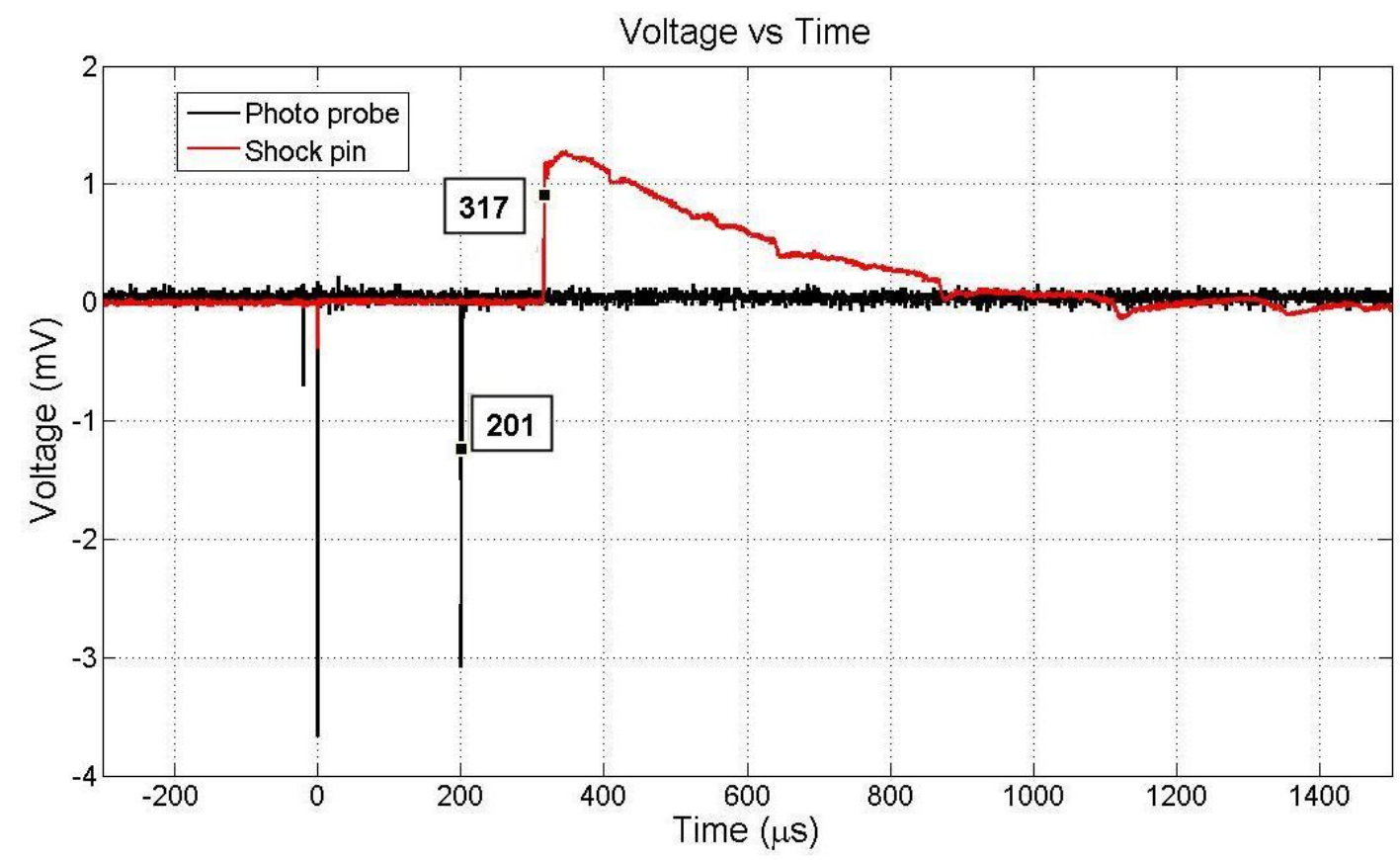


Fig. 3

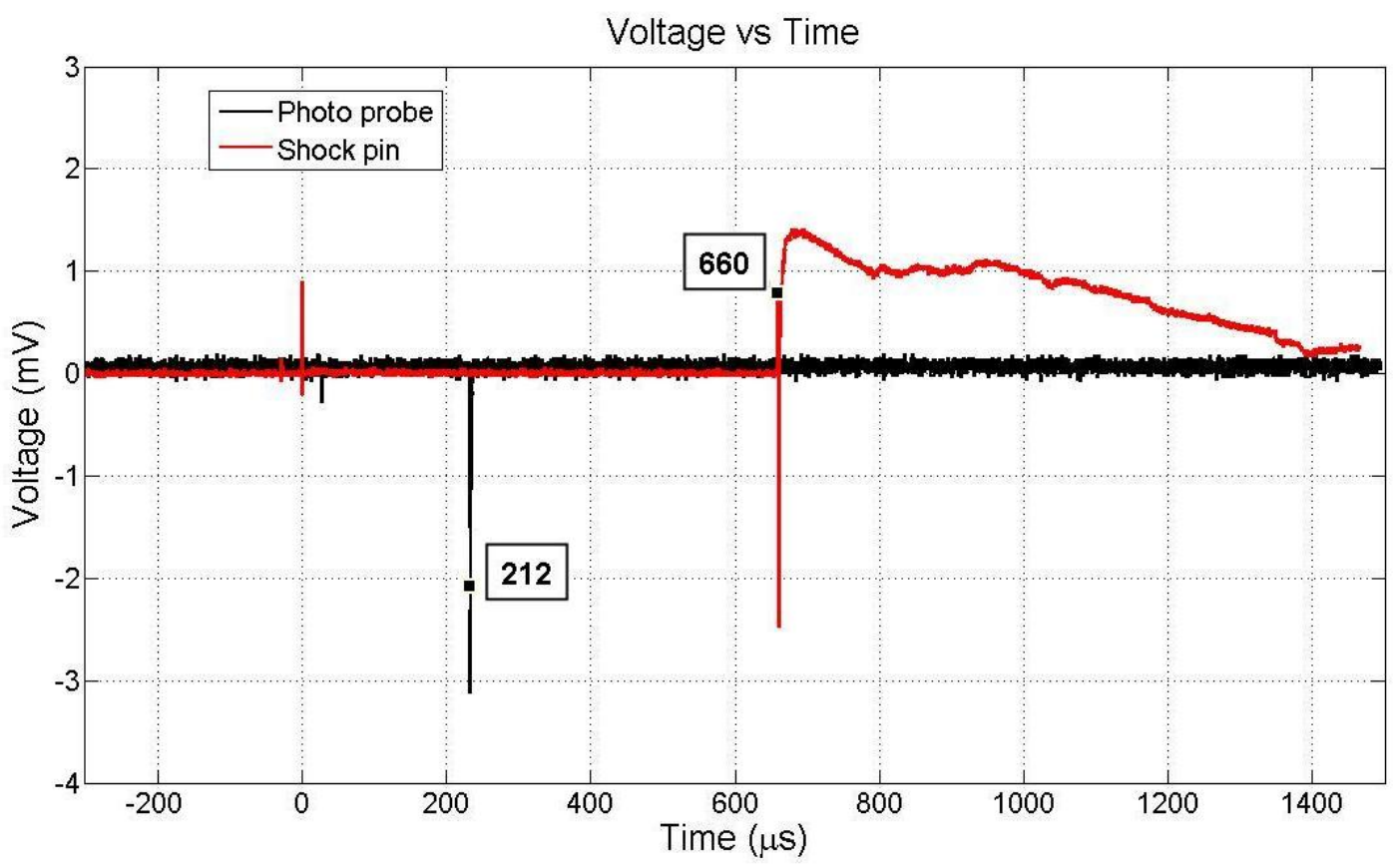


Fig. 4

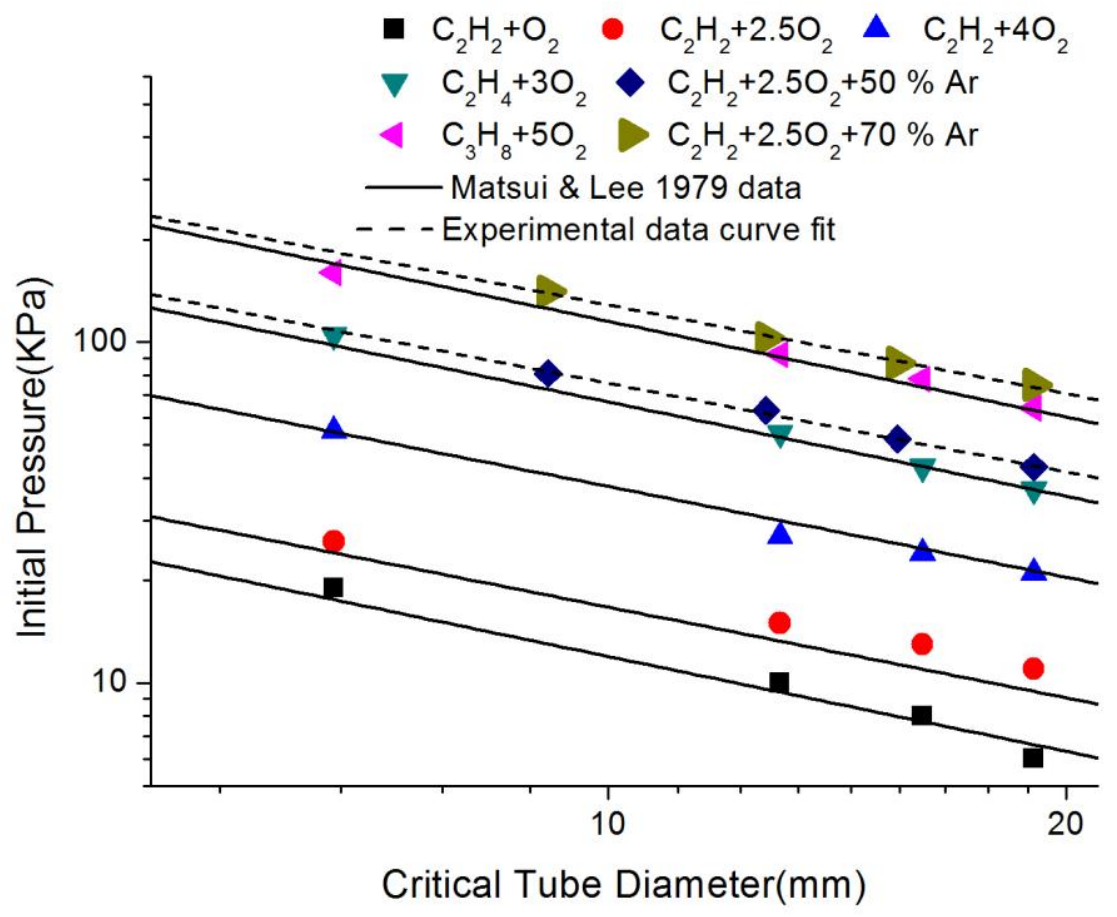


Fig. 5
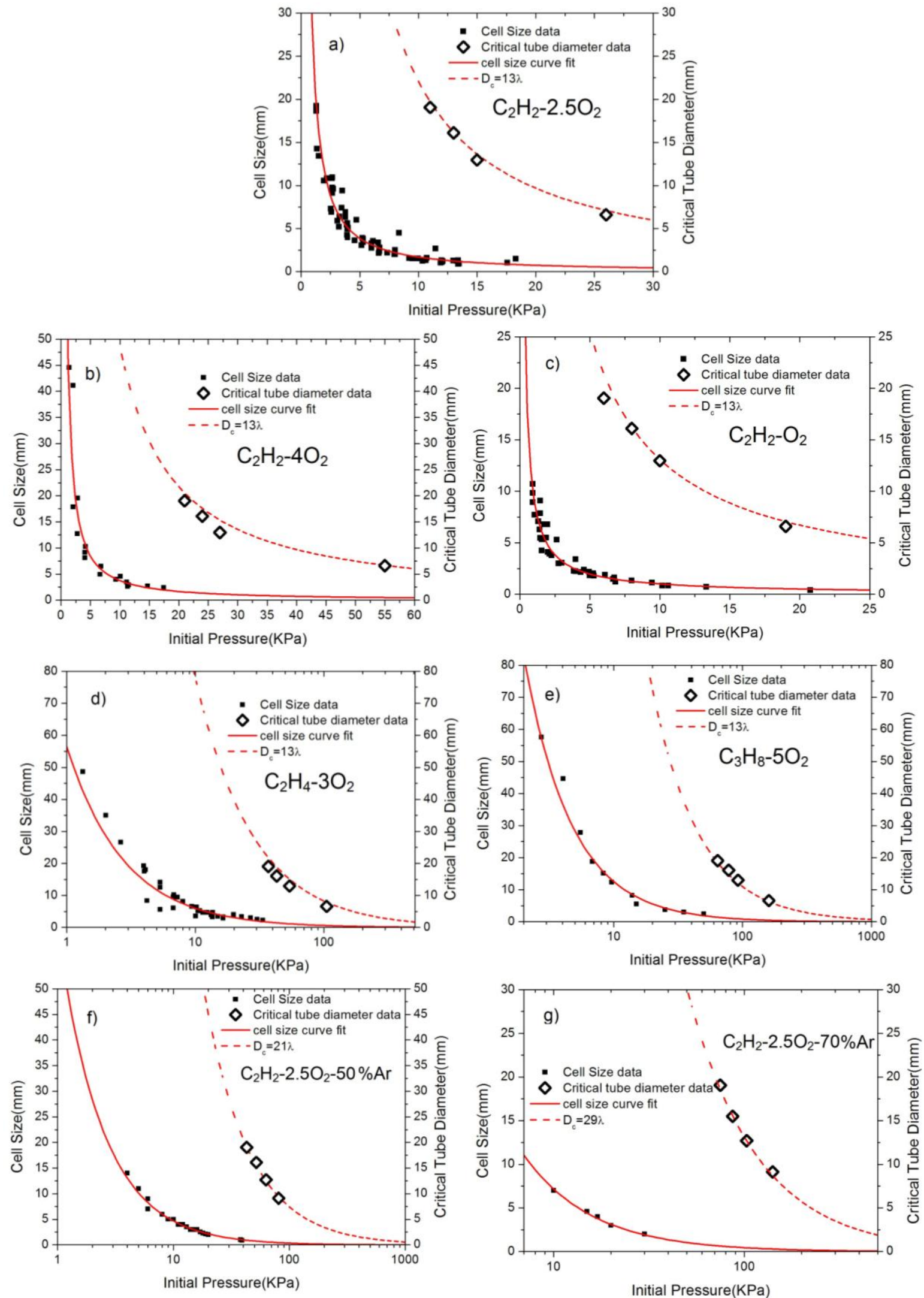


\section{Fig. 6}

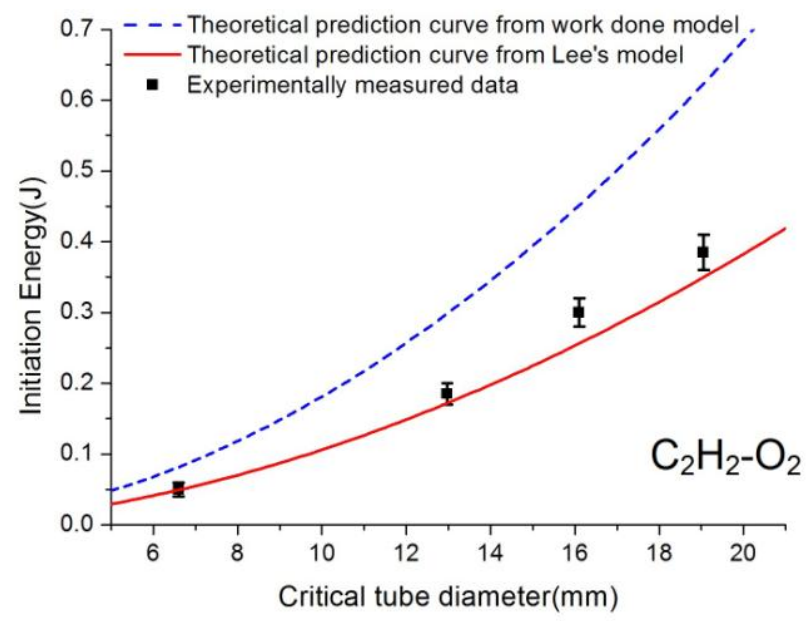

a)

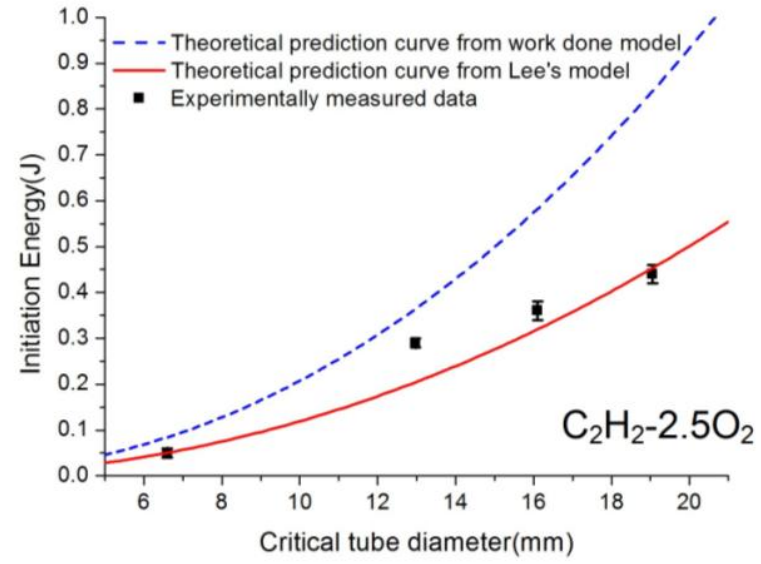

b)

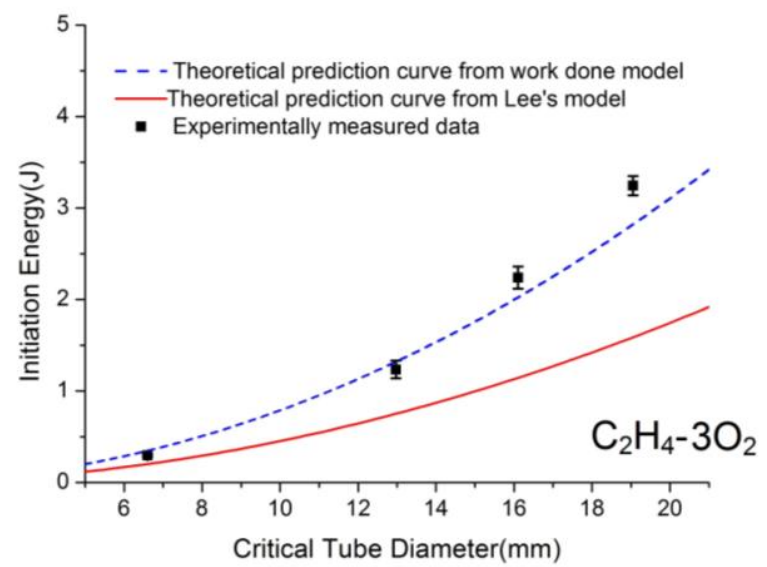

d)

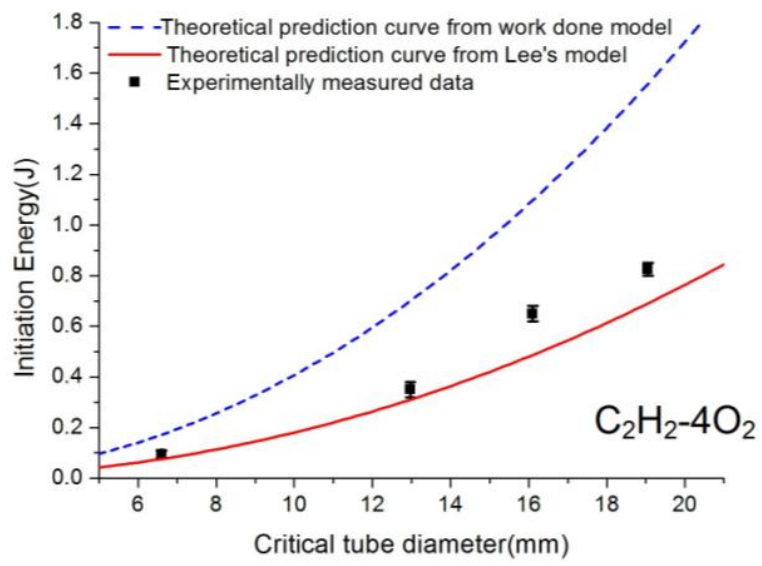

c)

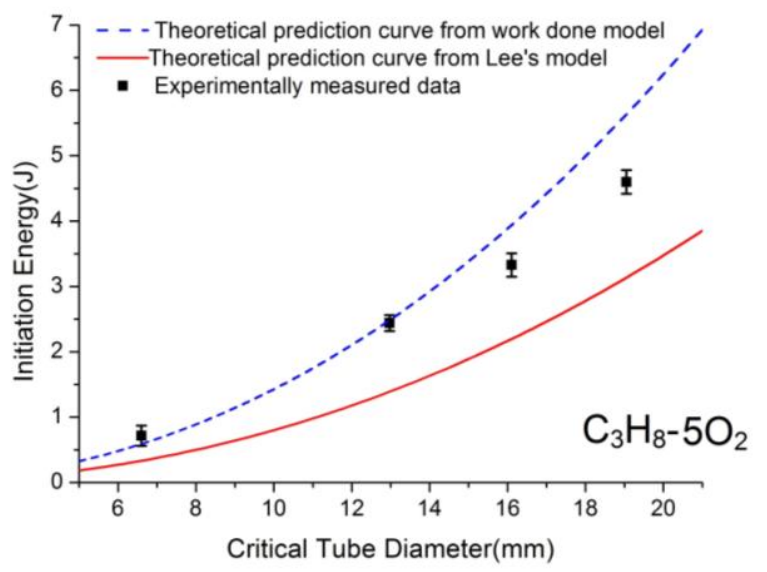

e) 
Fig. 7

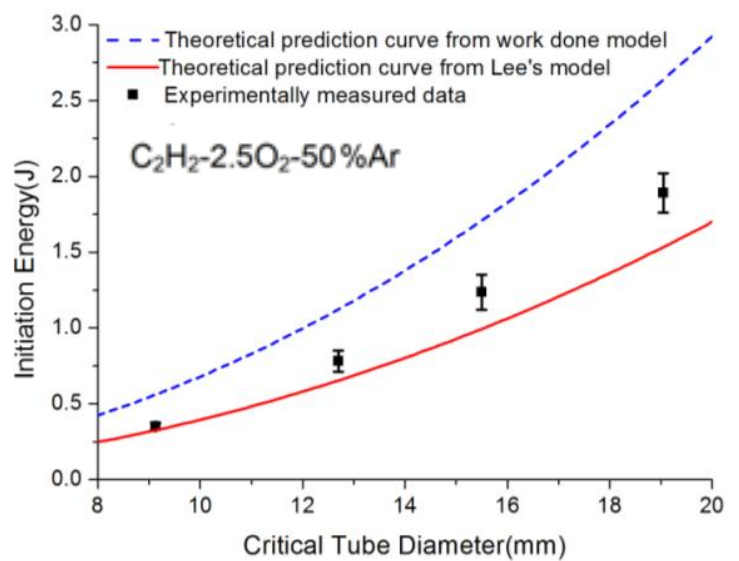

a)

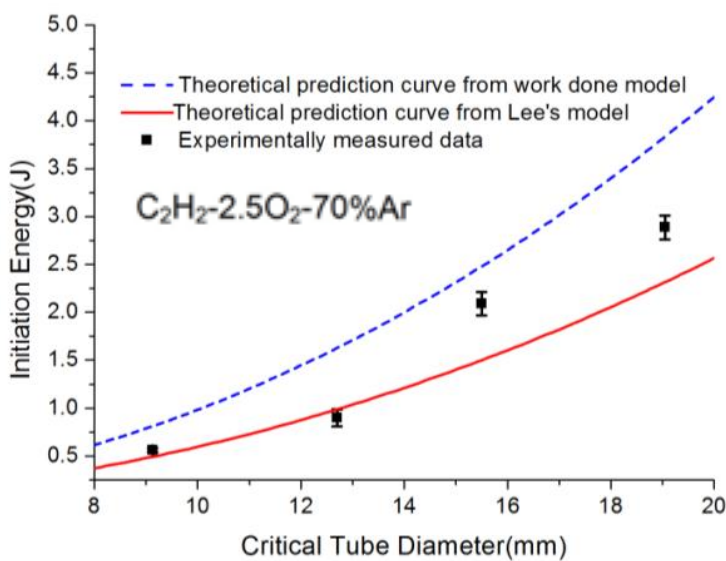

b) 